\title{
Bioética, assistência médica e justiça social
}

Ricardo Ney Oliveira Cobucci ${ }^{1}$, Lucélia Maria Carla Paulo da Silva Duarte ${ }^{2}$

\section{Resumo}

O Sistema Único de Saúde (SUS) brasileiro garante o acesso universal e integral aos serviços de saúde. Entretanto, na prática, o SUS não tem permitido que os cidadãos desfrutem de uma assistência com equidade, reforçando a dificuldade de se atingir a justiça social em uma sociedade tão desigual e injusta como a brasileira. A ética propõe a equidade como base para resolver as distorções na distribuição da saúde, possibilitando o acesso universal. Este artigo tem como objetivo precípuo discutir como a bioética pode auxiliar para que haja maior equidade na assistência médica em nosso país.

Palavras-chave: Bioética. Justiça social. Assistência à saúde.

\section{Resumen}

\section{Bioética, atención médica y la justicia social}

El Sistema Único de Salud (SUS) de Brasil garantiza el acceso universal e integral a servicios de salud. Sin embargo, en la práctica, el SUS no ha permitido a los ciudadanos a disfrutar de una asistencia con equidad, lo que aumenta la dificultad de lograr la justicia social en una sociedad tan desigual e injusta como la brasileña. La ética propone la equidad como base para resolver las distorsiones en la distribución de la salud, permitiendo el acceso universal. Este artículo tiene como principal objetivo discutir cómo la bioética puede ayudar a lograr una mayor equidad en la atención médica en Brasil.

Palabras-clave: Bioética. Justicia social. Prestación de atención a la salud.

\begin{abstract}
Bioethics, medical care and social justice

Brazilian's Unified Health System (SUS) ensures universal access to comprehensive health services. However, in practice SUS has not allowed citizens to enjoy a health care with equity, increasing the difficulty of achieving social justice in a society as unequal and unfair as the Brazilian. Ethics proposes equity as a basis for resolving the distortions in the distribution of health, enabling universal access. This article aims to discuss how bioethics can help for greater equity in health care in our country.

Key words: Bioethics. Social Justice. Delivery of health care.
\end{abstract}

1. Mestre rncobucci@hotmail.com 2. Mestranda lucelduart@yahoo.com.br - Universidade Federal do Rio Grande do Norte, Natal/ RN, Brasil.

\section{Correspondência}

Ricardo Ney Oliveira Cobucci - Rua Guilherme Tinoco, 1.236, apt 501 CEP 59022-070. Natal/RN, Brasil.

Declaram não haver conflito de interesse. 
O acesso igualitário, universal e integral aos serviços e ações de saúde, em termos legais no Brasil, é garantido pelo Sistema Único de Saúde (SUS). Entretanto, desde sua implementação tem sido difícil atingir a justiça social em uma sociedade tão desigual e injusta como a brasileira ${ }^{1}$. Essa não é uma dificuldade enfrentada apenas por nossa sociedade, pois existem desigualdades em saúde marcantes entre os países e dentro de um mesmo país. Por exemplo, a expectativa de vida varia de 34 anos em Sierra Leoa a 82 anos no Japão. Além disso, encontram-se diferenças de 20 anos na expectativa de vida entre populações ricas e pobres nos Estados Unidos da América (EUA) ${ }^{2}$.

Um importante ponto de partida para uma discussão dos determinantes sociais da saúde é a aplicação da teoria de John Rawls. Em A teoria da justiça este autor argumentou que a justiça requer a distribuição justa dos principais bens. Bens primários são alocados para indivíduos com base na igualdade de oportunidade justa, devido às desvantagens que os mesmos têm acumulado por meio da loteria natural da vida ${ }^{3}$. Na aplicação da teoria de Rawls para os determinantes sociais da saúde, Norman Daniels e cols. argumentam que justiça requer achatamento das desigualdades socioeconômicas de forma robusta, garantindo muito mais do que um mínimo de dignidade ${ }^{4}$.

Considerando que a justiça constitui princípio fundamental da bioética, o presente artigo tem como objetivo precípuo discutir como a bioética pode contribuir para tornar mais justa a assistência médica no Brasil.

\section{Dimensões da desigualdade em saúde}

As dimensões da desigualdade em saúde são explicadas pelas diferenças na distribuição, organização e utilização dos recursos em saúde. Além disso, um conjunto de fatores interligados às condições de saúde e adoecimento pode contribuir para uma maior desigualdade ${ }^{2,5}$.

Apesar da afirmação na Constituição brasileira e no Código de Ética Médica de que todo cidadão tem direito a cuidados médicos sem distinção de qualquer espécie, quer de raça, sexo, idade, condição social, nacionalidade, opinião política, religiosa ou de outra natureza, ou por ser portador de qualquer doença, infectocontagiosa ou não, a desigualdade persistente no acesso e uso de serviços de saúde em nosso país tem preocupado gestores, acadêmicos e legisladores, fomentando debates e pes- quisas com o objetivo de promover maior equidade 2,3,5. Tais discussões não se limitam apenas a explicar a desigualdade pelas diferenças existentes entre os grupos sociais, mas buscam incorporar nas análises conceituais a dimensão da justiça social. Dessa forma, tem sido possível caracterizar os diferentes tipos de desigualdade e propor, no campo político, a inclusão de valores éticos e morais capazes de tornar a sociedade mais igualitária ${ }^{5}$.

Nesse sentido, países mais desenvolvidos e mais pobres, com diferentes modelos de sistemas de saúde, voltam sua atenção para as desigualdades em saúde. É fato que entre esses países há diferenças, pois alguns apresentam assistência à saúde mais justa. Apesar disso, a preocupação de todos em diminuir a desigualdade deve ser exaltada, pois pode implicar na formulação e condução de políticas que proporcionem maior equidade $2,3,5$.

As desigualdades em saúde estão diretamente relacionadas com as desigualdades sociais. Portanto, ações efetivas que propiciam acesso mais igualitário aos serviços de saúde são importantes para diminuir as diferenças existentes entre os grupos sociais, relativas a adoecer e morrer ${ }^{5}$. Finalmente, para se implementar políticas equânimes deve-se contemplar três importantes campos na saúde: distribuição de recursos, oportunidades de acesso e utilização dos serviços ${ }^{5}$.

\section{Equidade na assistência médica}

A equidade é a base ética que deve guiar o processo decisório da alocação de recursos. A associação desse princípio com os da responsabilidade individual e pública e da justiça permite fazer valer o direito à saúde. Reconhecer as necessidades diferentes de sujeitos também diferentes para atingir direitos iguais é o caminho prático da ética que pode levar a um maior acesso à assistência médica ${ }^{6}$.

É necessário, portanto, estabelecer prioridades na assistência à saúde. Porém, ao optarmos por hierarquizar as necessidades em saúde com o objetivo de tornar mais justa a alocação de recursos, não podemos cometer erros como os ocorridos em Oregon e Seattle (EUA), onde, a partir de critérios altamente discutiveis, os alcoólatras foram colocados no final da lista de espera de transplante de fígado ${ }^{6}$. Nesse caso, houve uma preferência por pacientes candidatos a transplante que não consumiam álcool, pois consideraram mais importante privilegiar os demais, deixando claro o preconceito e a forma injusta de seleção. 
No Brasil e em muitos países, por razões exclusivamente econômicas, tem sido fundamental para o equilíbrio do sistema de saúde privar uma parcela da população do acesso aos avanços tecnológicos da medicina. São conhecidas, por exemplo, as diretrizes de muitos países que impedem a hemodiálise e transplantes de órgãos a pessoas com idade avançada ou portadoras de algumas doenças degenerativas ${ }^{7}$. Assim, qual a conduta ética frente à escassez de recursos na área da saúde? Esta parece ser uma questão que não tem resposta única e satisfatória ?

Outro dilema ético e comum na prática médica diária é como estabelecer critérios justos para decidir sobre qual paciente escolher diante de poucos recursos e da impossibilidade de oferecer assistência a todos. Pensadores utilitaristas como John Mill ${ }^{8}$ defendem que pacientes com melhores condições econômicas e, assim, com maior chance de sobreviver devem ser escolhidos no caso de haver apenas um rim para transplante. Em contraposição ao utilitarismo, existe a defesa da randomização na escolha feita por Childress ${ }^{9}$. Que método de escoIha adotar e quem deve ter a autoridade para tanto? Atualmente, na maioria dos países, cabe aos médicos essa decisão e muitos, apesar de treinados para priorizar a vida em detrimento da morte, sofrem por serem obrigados por um sistema desigual a optar sem ter a certeza se adotaram a escolha mais justa ${ }^{7}$.

Todo procedimento ético implica em escolhas, as quais definirão que pessoas serão prioritariamente beneficiadas ou não. A reflexão ética nos obriga a escolher com equilíbrio, pesando custos e benefícios, eficiência e eficácia, mas sem jamais esquecer os princípios da moralidade, prioridade e equidade ${ }^{6}$.

\section{Há equidade na assistência médica no Brasil?}

A América Latina e o Caribe passaram por reformas setoriais na década de 90 , patrocinadas e dirigidas por organismos internacionais como o Banco Mundial. Tais reformas apresentam alguns pontos em comum, como mudanças nas regras de financiamento, participação de agentes públicos e privados e separação das funções de provisão, regulação e financiamento ${ }^{10}$.

O Brasil não ficou totalmente imune a tais influências. Contudo, o SUS foi estruturado antes dessas reformas setoriais. O SUS representava uma proposta ampla reconhecida como Reforma Sanitária. A Reforma Sanitária brasileira pode ser considerada suprapartidária e independente, pois está vinculada às ideias de direitos humanos, de democratização da saúde e de cidadania ${ }^{11}$. Logo, esses organismos internacionais encontraram certa resistência e até mesmo uma oposição no Brasil, pois a Reforma Sanitária sempre defendeu o caráter universal das políticas de saúde. Entretanto, houve a aceitação de alguns pontos propostos pelas reformas setoriais, como a descentralização das ações em saúde e a segmentação do sistema de saúde ${ }^{11,12}$.

O país conseguiu manter os instrumentos legais que asseguram o direito à saúde, particularmente no que se refere à universalização da atenção à saúde, apesar dos constrangimentos impostos ao desenvolvimento das políticas de saúde. A política de saúde brasileira manteve sua abrangência, mas restrições aos gastos públicos e contradições aprofundaram a segmentação do sistema de saúde no país ${ }^{12,13}$. A distribuição das unidades de saúde é desigual entre as regiões e os estados, estando os estabelecimentos cadastrados mais concentrados na região Sudeste e nos municípios mais populosos ${ }^{14}$. Essa segmentação, explicitada pela assistência médica suplementar brasileira, acentua as desigualdades no consumo de serviços de saúde ${ }^{13}$. Atualmente, mais de 40 milhões de brasileiros buscam na saúde suplementar as soluções para seus problemas de saúde. Tal fato constitui-se no grande paradoxo do sistema de saúde brasileiro, que dessa forma exclui grande parte dos cidadãos de um sistema criado para ser universal. Além disso, o sistema de saúde suplementar é excludente com a criação de regras como doença preexistente e período de carência, fazendo com que seus usuários muitas vezes necessitem utilizar os serviços do SUS ${ }^{15}$.

A assistência médica privada, ao insinuar maior qualidade, agilidade e conforto aos pacientes, em comparação com o serviço público, acentua as desigualdades em saúde. Esse sistema voltado para uma atenção altamente especializada e orientada para a enfermidade prejudica o estabelecimento da equidade na assistência à saúde, haja vista que as pessoas não podem pagar por ele. Adicionalmente, a contratação não planejada de serviços privados pelo SUS e a oferta desordenada de assistência médica ambulatorial dificultam a realização do planejamento e da programação local em saúde e favorecem a concentração de atendimentos a uns indivíduos em detrimento de outros ${ }^{16}$.

\section{Bioética, assistência médica e justiça social}

Em matéria de assistência médica, os próximos anos deverão trazer um questionamento constante. 
Provavelmente, novas técnicas médicas não serão mais universalmente adotadas antes de uma avaliação custo-benefício. A busca será, cada vez mais, por um processo de racionalização. Porém, existem dificuldades entre os prestadores no sentido de entender o que é racionalização para um serviço de saúde. Caso racionalizar seja entendido como racionar, correremos o risco de oferecer serviços de saúde ineficientes e que acentuam as desigualdades ${ }^{17}$.

Para a resolução desse dilema da racionalização dos gastos precisamos superar dois obstáculos. 0 primeiro refere-se à profissão médica, que tem a responsabilidade da decisão e o poder de decidir além disso, o médico tem um papel muitas vezes conflituoso de conselheiro de seu paciente e de vendedor de serviços. O segundo, é a dificuldade de responder sobre a necessidade ou não da realização de exames complementares para elucidação diagnóstica e sobre qual o melhor tratamento. Se quisermos limitar os recursos destinados à prestação de serviços adequados, é preciso definir quais serão os níveis de assistência à saúde praticados. Mas em relação a isso são múltiplas as opiniões, mesmo as mais autorizadas. Em muitos casos, os médicos divergem sobre a natureza do melhor tratamento. Isto sem considerar a influência da relação médicopaciente, que muitas vezes passa do individual ao coletivo ${ }^{18}$.

A tomada de decisão na área médica é complexa e depende de variados fatores como considerações éticas, científicas, respeito à autonomia do doente, preferências do médico e influência dos familiares e da sociedade. Atualmente, a prática médica passa por uma crise quando o assunto se refere à tomada de decisão. De um lado, a pressão do crescimento econômico e do desenvolvimento tecnológico criando uma relação de consumo entre médicos e pacientes; de outro, o paciente, ser humano que busca a assistência médica como um direito fundamental e que deve ser oferecido pelo Estado. Em meio a eles o médico, despreparado para administrar tal conflito. Apenas reflexões promovidas por grupos multidisciplinares, propostas pela bioética, serão capazes de dirimir esses conflitos ou, ao menos, oferecer critérios para a tomada de decisão ${ }^{7,19,20}$.

Uma proposta interessante para auxiliar os profissionais de saúde é a da deliberação moral criada por Diego Gracia ${ }^{21}$. Segundo este autor, a deliberação decorre do reconhecimento e da aceitação da incomensurabilidade da realidade, dos quais resulta a necessidade de enriquecer a compreensão das coisas e dos fatos a partir da inclusão de diferentes visões e perspectivas. A deliberação considera impossível aplicar um raciocínio matemático às questões da vida prática, como a ética.

A deliberação moral consiste na consideração dos valores e deveres intervenientes em um fato concreto, a fim de se conduzir a situação por um caminho razoável, prudente e passível de realização. Sem incorrer em relativismos, a deliberação não visa encontrar a decisão ideal, certa ou que maximize os resultados, pois não se pauta pelas vertentes idealista, pragmática ou utilitarista, bastante usuais nas teorias e métodos de tomada de decisão ${ }^{21}$.

No que diz respeito à autonomia, direitos do cidadão, do médico e das instituições de saúde que precisam ser respeitados antes de qualquer decisão, Gutierrez ${ }^{22}$ considera que a conquista do respeito à autonomia é um fenômeno histórico bastante recente em nosso país, que pouco a pouco vem deslocando os princípios da beneficência e da não maleficência como prevalentes nas ações de assistência à saúde. Entretanto, afirma que o Código de Ética Médica limita a autonomia, que, assim como os direitos do cidadão, não pode ser considerada absolutamente, mas de acordo com as situações em que é exercida.

$O$ respeito pela autonomia da pessoa conjugase com o princípio da dignidade da natureza humana, aceitando que o ser humano é um fim em si mesmo, não somente um meio de satisfação de interesses de terceiros, comerciais, industriais ou dos próprios profissionais e serviços de saúde. Respeitar a pessoa autônoma pressupõe a aceitação do pluralismo ético-social, característico de nosso tempo. Porém, para Gutierrez, o respeito à autonomia não significa ser indiferente às circunstâncias, mas considerá-las com a objetividade possivel ${ }^{22}$.

Para finalizar, sublinha-se que para o efetivo alcance de sociedades mais equitativas são necessárias políticas públicas que superem o âmbito setorial e consigam, conjuntamente, diminuir as desigualdades atualmente existentes, como, por exemplo, o investimento no início do desenvolvimento infantil, programas de nutrição e de promoção à saúde, meIhorias na qualidade do ambiente de trabalho e reduções na desigualdade de renda, aliando-se a isso a eficiência, a eficácia e os princípios fundamentais da bioética. 


\section{Referências}

1. Carneiro Jr. N, Silveira C. Organização das práticas de atenção primária em saúde no contexto dos processos de exclusão/inclusão social. Cad Saúde Pública. 2003;19(6):1827-35.

2. Marmot M. Social determinants of health inequalities. Lancet. 2005;365(9464):1099-104.

3. Ruger JP. Ethics of the social determinants of health. Lancet. 2004;364(9439):1092-7.

4. Daniels N, Kennedy BP, Kawachi I. Why justice is good for our health: the social determinants of health inequalities. Daedalus. 1999;128(4):215-51.

5. Vianna AL, Fausto MCR, Lima LD. Política de saúde e equidade. São Paulo Perspec. 2003;17(1):5868.

6. Garrafa V, Oselka G, Diniz D. Saúde pública, bioética e equidade. Rev bioét (Impr.). 1997;5(1):2733.

7. Siqueira JE. A evoluçäo científica e tecnológica, o aumento dos custos em saúde e a questão da universalidade do acesso. Rev bioét (Impr.). 1997;5(1):41-8.

8. Mill JS. A liberdade/utilitarismo. São Paulo: Martins Fontes; 2000.

9. Beauchamp TL, Childress JF. Principles of biomedical ethics. Oxford: Oxford University Press; 2001.

10. Almeida C. Reforma de sistemas de servicios de salud y equidad en América Latina y el Caribe: algunas lecciones de los años 80 y 90. Cad Saúde Pública. 2002;18(4):905-25.

11. Almeida C. Saúde e equidade nas reformas contemporâneas. Saúde Debate. 2000;24(54):6-21.

12. Paim JS. Equidade e reforma em sistemas de serviços de saúde: o caso do SUS. Saúde Soc. 2006;15(2):34-46.

13. Almeida PF, Giovanella L, Mattos M. Sistema de saúde brasileiro: dilemas da universalização. Saúde Debate. 2002;26(61):137-54.

14. Saúde Brasil 2006: uma análise da desigualdade em saúde. Brasília: Ministério da Saúde, Secretaria de Vigilância em Saúde, Departamento de Análise de Situação em Saúde; 2006. (Série G. Estatísticas e Informações em Saúde).

15. Andrade EM, Andrade EO. O SUS e o direito à saúde do brasileiro: leitura de seus princípios, com ênfase na universalidade da cobertura. Rev bioét (Impr.). 2010;18(1):61-74.

16. Costa NR. Política social e ajuste macroeconômico. Cad Saúde Pública. 2002;18 (Supp.):13-21.

17. Noronha JC, Soares LT. A política de saúde no Brasil nos anos 90. Ciênc Saúde Coletiva. 2001;6(2):445-50.

18. Starfield B. Atenção primária: equilíbrio entre necessidades de saúde, serviços e tecnologia. Brasília: Unesco/Ministério da Saúde; 2002.

19. Zucchi P, Del Nero C, Malik AM. Gastos em saúde: os fatores que agem na demanda e na oferta dos serviços de saúde. Saúde Soc. 2000;9(1/2):127-50.

20. Mcpherson P. Variations entre pays des pratiques médicales. OCDE Etudes de politique sociale. 1990;7:17-30.

21. Gracia D. Moral deliberation: the role of methodologies in clinical ethics. Med Health Care Philos. 2001;4(2):223-32.

22. Gutierrez PL. Reflexões sobre autonomia e direitos do cidadão, do médico e das instituições de saúde: do bem individual ao bem coletivo. In: Constantino CF, Barros JCR, Hirschheimer MRH, editores. Cuidando de crianças e adolescentes sob o olhar da ética e da bioética. Rio de Janeiro: Atheneu; 2009, p. 347-53.

Participação dos autores no artigo

Ambos os autores colaboraram integralmente na redação do artigo.

Recebido: 2.11.2011

Revisado: 17.8 .2012

Aprovado: 15. 2.2013 